Meta

Journal des traducteurs

Translators' Journal

\title{
Réflexions sur les études des problèmes fondamentaux de la traduction
}

\section{$\mathrm{Xu}$ Jun}

Volume 44, numéro 1, mars 1999

Théorie et pratique de la traduction en Chine

The Theory and Practice of Translation in China

URI : https://id.erudit.org/iderudit/004625ar

DOI : https://doi.org/10.7202/004625ar

Aller au sommaire du numéro

Éditeur(s)

Les Presses de l'Université de Montréal

ISSN

0026-0452 (imprimé)

1492-1421 (numérique)

Découvrir la revue

Citer cet article

Jun, X. (1999). Réflexions sur les études des problèmes fondamentaux de la traduction. Meta, 44(1), 44-60. https://doi.org/10.7202/004625ar
Résumé de l'article

Cet article étudie les problèmes fondamentaux qui se posent aux traducteurs de tous lestemps et de tous les pays. Par une comparaison succincte des réflexions théoriqueschinoises et françaises sur la traduction, l'auteur souligne la similitude et l'oppositiondualiste entre traduction libre et littérale qui se perpétue à travers le temps et l'espace. Ledébat sur la retraduction du Rouge et le Noir en Chine en offre un parfait exemple. Poursortir de cette opposition dualiste dans les études de traduction, l'auteur propose uneétude des niveaux de traduction, en analysant les éléments interdépendants et interactifsdes trois niveaux de la traduction : le niveau de pensée, le niveau sémantique et le niveauesthétique. Le but est donc d'ouvrir un domaine ou un nouvel angle de réflexion sur lanature et le processus de la traduction, et d'en mesurer objectivement la complexité et latâche pour avoir une conscience plus juste des problèmes fondamentaux du traduire.
Ce document est protégé par la loi sur le droit d'auteur. L'utilisation des services d’Érudit (y compris la reproduction) est assujettie à sa politique d'utilisation que vous pouvez consulter en ligne.

https://apropos.erudit.org/fr/usagers/politique-dutilisation/ 


\title{
Réflexions sur les études des problèmes fondamentaux de la traduction
}

\author{
$x \mathbf{u}$ jun \\ Université de Nanjing, \\ Nanjing, Chine
}

\section{RÉSUMÉ}

Cet article étudie les problèmes fondamentaux qui se posent aux traducteurs de tous les temps et de tous les pays. Par une comparaison succincte des réflexions théoriques chinoises et françaises sur la traduction, l'auteur souligne la similitude et l'opposition dualiste entre traduction libre et littérale qui se perpétue à travers le temps et l'espace. Le débat sur la retraduction du Rouge et le Noir en Chine en offre un parfait exemple. Pour sortir de cette opposition dualiste dans les études de traduction, l'auteur propose une étude des niveaux de traduction, en analysant les éléments interdépendants et interactifs des trois niveaux de la traduction: le niveau de pensée, le niveau sémantique et le niveau esthétique. Le but est donc d'ouvrir un domaine ou un nouvel angle de réflexion sur la nature et le processus de la traduction, et d'en mesurer objectivement la complexité et la tâche pour avoir une conscience plus juste des problèmes fondamentaux du traduire.

\begin{abstract}
The author looks at some of the fundamental problems faced by translators. Through a comparison of French and Chinese translation theories, he focuses on the similarity and dichotomy between free translation and literal translation. A discussion of the (re)translation of Stendhal's Le Rouge et le Noir in China serves as a perfect illustrative example. To move away from this dichotomy in translation studies, the author proposes a study of levels of translation (with analysis of interdependent and interactive elements): the conceptual level (thought), the semantic level and the esthetic level. The aim is to adopt a new approach to discussions on the nature of translation and the processes involved and to better assess the complexity of the translator's task.
\end{abstract}

Lorsqu'on aborde une traduction, elle nous paraît toujours trop compliquée, car les problèmes qu'elle soulève peuvent se poser à tous les niveaux et sont d'aspects différents. Pourtant, si l'on jette un regard rétrospectif sur la pratique traduisante et les réflexions des traducteurs chinois et étrangers, on s'aperçoit que sous cette apparence complexe et contradictoire, certains problèmes essentiels posés à la traduction se perpétuent à travers les siècles. Cet article essaye de dégager, à partir des réflexions théoriques chinoises et françaises, quelques constats et propose une vue synthétique sur les études des problèmes fondamentaux de la traduction.

\section{LA FIDÉLITÉ, PROBLÈME-CLÉ DE LA TRADUCTION}

Quand je parle des problèmes fondamentaux de la traduction, je renvoie tout de suite à Antoine Berman (1984: 12) : 
La constitution d'une histoire de la traduction est la première tâche d'une théorie moderne de la traduction. À toute modernité appartient, non un regard passéiste, mais un mouvement de rétrospection qui est une saisie de soi.

En effet, j'entends par problèmes fondamentaux ceux qui se posent tout au long de I'histoire de la traduction et auxquels tous les traducteurs devraient apporter une réponse. Ainsi, pour cerner ces problèmes, un regard rétrospectif sur I'histoire de la traduction paraît nécessaire. Les ouvrages portant sur l'histoire de la traduction que nous avons pu lire en français ou en anglais sont: Les Belles Infidèles de Georges Mounin, La Traduction dans le monde moderne et Les Grands Traducteurs français d'Edmond Cary, The True Interpreter. A H istory of Translation Theory and Practice in the West de L.G. Kelly, Petite Histoire de la traduction en Occident de Henri Van Hoof, De Cicéron à Benjamin, traducteurs, traductions et réflexions de $\mathrm{M}$ ichel Ballard, etc. En Chine, parmi les réflexions historiques sur la traduction, qu'il nous soit permis de signaler le Précis de l'histoire de la traduction en Chine, de son origine jusqu'en 1919 de Ma Zuyi, la Petite Histoire de la traduction en Occident de Tan Zaixi et I'H istoire des études théoriques de la traduction en Chine de Chen Fukang. Me basant sur ces documents et élaborant une étude comparée des réflexions théoriques sur la traduction, j'ai essayé de situer les problèmes posés à la traduction sous trois aspects (volonté, réalité et rationalité), ce qui m'a permis de cerner certains invariants des réflexions historiques sur la traduction et de constater que tout au long de l'histoire, les problèmes fondamentaux restent toujours les mêmes, seules les solutions varient selon le temps, l'espace et les relations que le sujet traduisant entretient avec le texte d'origine et le lecteur. Je ne prétends pas recenser ici tous les problèmes fondamentaux que les praticiens et théoriciens de la traduction ont abordés, je me contente seulement de montrer par une approche comparative la similitude des réflexions chinoises et françaises centrées sur les problèmes qui dérivent de la notion de fidélité, et de réfléchir sur l'étude de ces problèmes.

Dans Les Grands Traducteurs français, Edmond Cary place le problème de la fidélité au centre du débat éternel en indiquant (1963: 21) :

La fidélité à l'original, principe invariablement proclamé par tous les traducteurs et qui n'en mène pas moins aux plus étonnantes contradictions, est sans doute la notion centrale du débat autour de la traduction et dont chaque siècle exhume à nouveau le dossier.

Au sujet de la fidélité, je suis bien conscient que cette notion a suscité, et suscite encore, bien des controverses, d'autant plus qu'elle est «très souvent comprise comme synonyme de "littéralité" par opposition à "traduction libre" ». Traduction fidèle, mais à quoi ? La question se pose tout de suite, commel'a fait Georges M ounin. Bien que controver sée, cette notion traduit dans une large mesure le souci d'une prise de position par le traducteur dans ses relations avec l'original et avec le lecteur, et définit l'existence du lien souhaité entre l'original et sa traduction. À lire les documents historiques sur les réponses apportées par certains traducteurs et théoriciens à cette notion-clé de la théorie de la traduction, on est frappé par une similitude étonnante entre l'histoire de la traduction chinoise et occidentale. Du problème-clé de la traduction qu'est la fidélité dérive toute une série de binômes, tels que la traduction littérale et la traduction libre, la traduction des mots et la traduction du sens, la fidélité à la forme et la fidélité au fond, la fidélité au corps et la fidélité à l'âme, 
l'imitation et la recréation, la fidélité et la trahison, l'exotisme et la naturalisation, la traduction sourcière et cibliste, le vrai et le beau, l'exactitude et la beauté, la possibilité et l'impossibilité, etc. La formulation de ces problèmes est presque la même en Occident et en Chine. Remontons tout d'abord à la source de la théorie occidentale de la traduction. Voici un passage très connu du M eilleur genre d'orateur de Cicéron :

J'ai mis en latin les deux plus célèbres discours des deux Attiques les plus éloquents, Eschine et Démosthène, discours dont l'un répond à l'autre; je les ai mis en latin, non pas en traducteur mais en orateur ; les pensées restent les mêmes, ainsi que leur tour et comme leurs figures; les mots sont conformes à l'usage de notre langue. Je n'ai pas cru nécessaire de rendre mot pour mot, c'est le ton et la valeur des expressions dans leur ensemble que j'ai gardés. J'ai cru qu'il me fallait payer le lecteur non pas en comptant pièce par pièce, mais pour ainsi dire en pesant la somme en bloc.

II serait trop long d'analyser à fond les facteurs impliqués dans la traduction, pris en considération par Cicéron, et d'établir les relations entre le but ou l'objectif fixé et les méthodes de traduction, mais je voudrais simplement souligner l'importance de ces lignes car, dans I'histoire de la traduction en Occident, on s'en sert très souvent pour justifier une façon de traduire, dite libre, sans se soucier de la fidélité aux formes de I'original, comme l'a indiqué M ichel Ballard (1995). C'est ainsi qu'en France, Étienne Dolet a formulé cinq principes de la traduction (1540), en signalant clairement:

qu'en traduisant il ne le fault pas asservir jusqu'à là que l'on rende mot pour mot, [...] il te fault garder d'usurper mots très approchants du latin [et] l'observation des nombres oratoires...

Jacques Amyot va plus loin qu'Étienne D olet, il définit sa position en ces termes (dans Cary 1963: 17) :

L'office d'un propre traducteur [... ] ne gist pas seulement à rendre fidèlement la sentence de son autheur, mais aussi à adombrer la forme du style et manière de parler d'iceluy.

C'est ainsi que Nicolas Perrot, sieur D'Ablancourt, s'est appuyé sur la manière de Térence et de Cicéron et se croyait dans son droit de modifier ou de censurer le texte au nom du bon goût. Dans sa préface à l'H istoire véritable de Lucien, Perrot D'Ablancourt a expliqué ce qu'il avait fait du texte d'origine (v. Cary 1963) :

J'y ai retranché ce qu'il y avait de plus sale et adouci en quelques endroits ce qui était trop libre [...] sans parler des vieilles fables trop rabattues, de proverbes, d'exemples et de comparaisons surannées, qui feraient à présent un effet tout contraire à son dessein ; car il s'agit ici de galanterie et non pas d'érudition.

II dit plus loin (dans Ballard 1995: 172) :

Je ne m'attache donc pas toujours aux paroles ni aux pensées de cet auteur, et demeurant dans son but, j'agence les choses à notre air et à notre façon. Les divers temps veulent non seulement des paroles, mais des pensées différentes.

C'est ainsi peut-être que dans les dernières années de notre siècle, «la traduction en français de La Plaisanterie s'apparentait davantage à un travail de réécriture, le traducteur ayant ajouté au gré de son inspiration quelques métaphores, formules alambiquées et tournures qui se voulaient amusantes», de telle sorte que Kundera s'est révolté contre cette version française: «Aujourd'hui encore, j'en suis malheu- 
reux. Penser que pendant douze ans, dans de nombreuses réimpressions, La Plaisanterie s'exhibait en France dans cet affublement ${ }^{1}$. »

Je ne peux citer ici toutes les réflexions qui peuvent justifier la traduction dite libre. $M$ ais il est peut-être nécessaire de faire entendre d'autres voix qui combattent l'infidélité. Écoutons d'abord la voix de Bacchet de M éziriac qui insistait sur l'importance de la fidélité et de l'exactitude. II dit (v. Ballard 1995: 164-165) :

La beauté du langage ne suffit pas pour faire estimer une traduction excellente. II n'y a personne qui n'avoue que la qualité la plus essentielle à un bon traducteur, c'est la fidélité...

Selon lui, pour faire unetraduction fidèle, il faut observer trois points: «qu'il n'ajoute rien à ce que dit son Auteur, qu'il n'en retranche rien, et qu'il n'y rapporte aucun changement qui puissent altérer le sens» (v. Ballard 1995: 164-165).

Après lui, Pierre-Daniel Huet a publié en novembre 1661 son traité De Interpretatione en s'opposant à la traduction libre qui cherche à modifier et à embellir la traduction au nom du bon goût et de la recréation. Pierre-Daniel Huet demande que I'on respecte l'original (v. Ballard 1995: 184-185) :

Pour bien traduire, il faut d'abord s'attacher à la pensée de l'auteur et ensuite aux mots eux-mêmes autant que le permet le génie des deux langues, afin de rendre l'originalité du texte en toutes ses parties, sans restriction ni exagération. [Et] pour l'Écriture et les pères, la traduction littérale est seule admise, la dignité du sujet exige que ces livres soient rendus d'une manière très simple.

Pas entièrement étouffée aux $x \mathrm{v}^{\mathrm{i}}{ }^{\mathrm{e}}$ et $\mathrm{xv} \mathrm{ii}^{\mathrm{e}}$ siècles en France, la voix de la traduction littérale se fait entendre beaucoup plus au xix ${ }^{e}$ siècle et dans le nôtre. La France a ainsi connu la traduction photographique du Faust par Nerval, le calque à la vitre du Paradis perdu par Chateaubriand et la littéralité la plus scrupuleuse observée par Leconte de Lisle quand il traduisait I'Iliade et l'O dyssée. Dans le dossier «Faut-il tout retraduire?» (Lire, $n^{\circ} 252$ ), Catherine Argand nous a tracé un itinéraire parcouru en France par le traduire depuis le xvii i siècle:

Au xviije siècle, les Français acharnés à défendre le beau parler (et les bonnes mœurs) taillaient à cœur en veux-tu dans les textes des romantiques anglais. Au début de notre siècle, on francise encore à outrance, sous prétexte de ne pas vouloir choquer ou dépayser le public. Depuis, l'authenticité est devenue un dogme. La traduction se doit de maintenir l'étrangeté du texte venu d'ailleurs tout en transposant les références dans la langue du lecteur.

En Chine, I'histoire du traduire ne suit pas le même ordre chronologique qu'en France. Mais les voix de la traduction libre et «directe» (comme synonyme de litté rale) sont aussi présentes qu'en France à travers les siècles. L'histoire de la traduction chinoise peut se diviser grosso modo en quatre périodes principales: traduction des soutras, traduction à l'époque des dynasties des Ming et des Qing, traduction du début de notre siècle et traduction depuis 1949. Quand commence vraiment la traduction des soutras? Les historiens ont des points de vue différents. $M$ ais, selon les documents historiques, on peut affirmer que la traduction des soutras en Chine peut remonter au moins en 148 ap. J.-C. En ce qui concerne les réflexions théoriques sur la traduction, la «Préface» à la traduction des Canons bouddhiques de Zhiqian est considérée comme le premier traité sur la traduction. Dans cette préface, on se réfère au 
dilemme très connu posé par Laozi, un des plus grands philosophes chinois: la parole belle n'est pas fidèle et la parole fidèle n'est pas belle. Pour rendre fidèlement les Canons bouddhiques, Zhiqian a posé un principe: être fidèle à l'original et ne point l'embellir. Ce qui nous fait penser tout de suite aux «belles infidèles» françaises qui ont partout leurs cousines. En Chine, dès les premières réflexions sur la traduction, on est pleinement conscient des difficultés linguistiques et ontologiques de la traduction. Après Zhiqian, à l'époque de la dynastie de Dong Jin, Dao An évoque en termes très précis cinq cas où l'on risque de perdre (ou trahir) I'original, à savoir renverser l'ordre des mots de l'original, l'embellir, le simplifier, en retrancher ce qui est répétitif et en modifier l'articulation. II s'oppose ainsi à la façon de traduire qui pourrait trahir l'original en cherchant à l'embellir. Comme l'on vient de le montrer, la fidélité à I'original est donc tenue pour un critère primordial de traduction dont se réclament non seulement les traducteurs religieux, mais aussi les traducteurs littéraires. À l'époque de la dynastie des Q ing, un traducteur nommé Wei Xiangqian (魏象乾) a tracé la vraie voie à suivre aux traducteurs; elle consiste à bien entendre la pensée du texte d'origine, à reproduire son style, à transmettre son souffle et à réexprimer son esprit et, en même temps, à ne rien ajouter, à ne rien retrancher et à ne rien changer ou renverser. C'est presque la même voix que Bacchet de M éziriac.

Ce qui est paradoxal, c'est que si l'on est absolument fidèle au texte d'origine, on risque au contraire de le déformer. Dans I'histoire de la traduction chinoise, nombreux sont les traducteurs qui essayent de se libérer des contraintes linguistiques du texte d'origine et de traduire de façon intelligente pour faciliter la lecture. Parmi les traducteurs des soutras, Kumarajiva (鸭摩罗什 344-413) a été assez libre pour ajouter ou retrancher ce qu'il trouvait nécessaire. II a même déclaré que, parmi plus de cinq cents volumes traduits, un seul n'a pas connu de retranchement. Pour lui, il importe de modifier le texte d'origine pour transmettre son esprit. Il était contre la traduction trop directe (dans Chen 1992: 26) :

Dans la traduction du sanscrit en chinois, si l'on perd ses belles couleurs d'expression, on risque de faire manger ce qu'on avait mâché, ce qui altérait non seulement le bon goût, mais aussi ferait vomir, car le style n'est pas du tout rendu, bien que le sens soit transmis en gros.

改梵为秦, 失其藻蔚, 虽得大意, 殊隔文体, 有似嚼饭与人, 非徒失味, 乃令呕秽 也

Après Kumarajiva, les tenants de la traduction libre développent peu à peu leurs théories et soutiennent qu'à calquer la forme de l'original, la traduction directe mène plutôt à l'illisibilité. Pour que la traduction soit acceptable par les lecteurs, il faut, au contraire, garder une certaine liberté à l'égard de l'original et essayer de rendre de façon expressive et élégante l'esprit de l'original. C'est ainsi que, dans la dynastie des Tsing, on a connu «la fidélité, l'expressivité et l'élégance», trois principes formulés par Yanfu, qui sont presque identiques aux idées de Pierre Coustel qui insiste, dans son ouvrage intitulé Les Règles de l'éducation des enfants, publié en 1687, sur la fidélité au sens, la clarté et l'élégance de l'expression:

Quand je dis qu'il faut être fidèle dans la traduction, ma pensée n'est pas qu'il faille scrupuleusement s'assujettir à toutes ses paroles et le traduire mot pour mot, mais je dis qu'il suffit de le traduire sens pour sens; c'est-à-dire qu'il suffit d'exprimer en français, 
par exemple, tout le sens qui est dans le latin ou le grec, sans s'attacher servilement ni à

l'ordre des mots, ni aux tours qui sont propres et naturels à chaque langue.

En ce qui concerne les relations entre le sens et les paroles, Coustel emploie une métaphore (v. Ballard 1995: 182) :

Le sens est comme l'âme du discours, et les paroles n'en sont que comme le corps. Ainsi une traduction toute littérale est comme un corps sans âme, parce que le corps est d'une langue et l'âme d'une autre.

Cette métaphore est bien familière aux théoriciens chinois, car Fu Lei, un des plus grands traducteurs littéraires chinois, a comparé la traduction à la peinture en disant que ce qu'on cherche, dans la traduction, n'est pas tant la ressemblance de l'âme que celle du corps. Pour lui,

rien n'empêche de penser que la traduction idéale serait une création de l'œuvre en chinois. Le sens complet et l'esprit de l'original ainsi que la clarté de la traduction pourraient alors être pris également en considération².

Comparons ce passage à ce qu'a formulé Coustel en la matière: qu'on puisse dire que si l'auteur sur lequel on travaille avait, par exemple, écrit en notre langue, ce serait ainsi qu'il aurait parlé...

Nous avons touché ici une notion très chère à la traduction littéraire: traduire, c'est recréer en langue cible. Avec cette notion de recréation, on passe sous silence l'opposition dualiste entre la fidélité et la trahison, la forme et le sens, etc. Au nom de la recréation, les traducteurs ont acquis une liberté plus ou moins grande, en se fixant un but et en modifiant selon le but fixéleurs relations avec l'auteur et le lecteur. Mais, de nos jours, les voix sont loin d'être unanimes. Pour certains, traduire une œuvre c'est essayer de s'approcher d'elle, c'est-à-dire aller vers le texte d'origine. Pour eux, on devrait respecter l'original, établir des relations d'égalité entre l'auteur et le traducteur. Pour d'autres, traduire une œuvre c'est rivaliser avec elle, et si I'on rivalise, il n'est plus question d'être fidèle à l'original ni de le suivre pas à pas, mais tout au contraire, il faut essayer de le dépasser, en mettant en valeur les avantages de la langue d'arrivée. Depuis quelque temps, la Chine connaît une vogue sans précé dent de retraduction littéraire. Par exemple, en l'espace de quelques années, on a vu paraître une dizaine de versions chinoises du Rouge et le N oir de Stendhal. Et un débat acharné s'est engagé, en 1995, à l'échelle nationale, sur la traduction en chinois de cet ouvrage, tout comme, en France, la traduction radicalement nouvelle de Dostoïevski par André Markowicz a semé une «belle pagaille», il y a trois ans. II faut dire que le débat sur la traduction et la retraduction du roman de Stendhal est plutôt positif et significatif car, avec des versions différentes en chinois, s'est posée toute une série de problèmes fondamentaux dérivant de la notion de fidélité que nous venons d'évoquer et de la simple comparaison des réflexions chinoises et françaises sur la traduction.

\section{UN DÉBAT SIGNIFICATIF}

La retraduction n'est pas un phénomène particulier à la Chine: il est universel. Les 36 versions de l'Énéide que la France a connues jusqu'ici en sont un bon exemple. II n'y a donc rien d'étonnant que le roman de Stendhal connaisse 15 ou 20 versions 
chinoises. Ce qui nous intéresse, c'est que ces retraductions peuvent nous fournir matière à réflexion, en soulevant les problèmes fondamentaux relatifs à la traduction. En effet, dans le débat sur la retraduction de cette œuvre, qui a eu lieu en 1995, non seulement les voix des traducteurs étaient présentes mais aussi celles des lecteurs, des critiques et des théoriciens de la traduction. Nous avons pu les entendre, soit dans les préfaces ou les postfaces des traductions, soit dans la correspondance qu'échangeaient traducteurs et critiques dans les revues ou les journaux où les critiques et les traducteurs s'affrontaient, dans les interviews ou dans des discussions intéressées, et surtout dans les réponses au questionnaire du Centre de traduction de l'Université de Nanjing élaboré en collaboration avec le journal hebdomadaire de Shanghai Lire.

Ce débat sur la traduction était, selon les critiques, un des plus grands évé nements dans le milieu de la traduction littéraire depuis un demi-siècle. II était inévitable et significatif : inévitable, parce quela retraduction s'inscrivait dans le cadre d'une grande vogue de retraduction, sans précédent dans l'histoire chinoise, qui présente un vif intérêt d'études pour des raisons littéraires, culturelles, sociologiques, économiques et historiques. Les 15 versions chinoises, dont une dizaine ont vu le jour ces dernières années, présentent des différences à divers niveaux: lexical, sémantique, stylistique. Les traducteurs célèbres et expérimentés comme Zhao Ruihong, le premier traducteur chinois du Rouge et le Noir, Luo Yujun, Hao Yun, Wen Jiasi, Luo Xinzhang, Xu Yuanchong et Guo Hongan, ont chacun leurs propres réflexions sur la traduction, aussi variées qu'opposées, qui influencent nécessairement leur pratique. Le débat était significatif, parce que cette vogue de retraduction a beaucoup contribué aux échanges culturels et a répondu aux goûts variés des lecteurs à différents niveaux, mais en même temps, on peut aussi constater certains effets négatifs sous cette prospérité apparente. Des lecteurs commencent à se plaindre de «l'embarras du choix», et de la mauvaise qualité de certaines traductions qui sont pleines d'erreurs ou de déformations, et portent même des traces de plagiat, tandis que des critiques, en comparant les versions représentatives, réfléchissaient sur les raisons de leurs différences. Quelle attitude le public en général adopte-t-il alors visà-vis de la floraison de versions différentes d'une même œuvre littérai re étrangère? Quel est le goût des lecteurs d'aujourd'hui ? Quelle œuvre préfèrent-ils parmi ces versions représentatives? Que pensent les lecteurs des réflexions de ces traducteurs? C'était justement pour éclaircir ces points que le Centre de recherche en traduction a mené, au mois d'avril 1995, cette enquête auprès des lecteurs chinois en collaboration avec le Comité de rédaction de Lire. Voici les neuf principales questions posées aux lecteurs:

1. Le Rouge et le Noir, plusieurs fois retraduit, compte aujourd'hui plus de dix versions chinoises. Que pensez-vous de ce phénomène?

2. La traduction littéraire devrait-elle contribuer aux échanges culturels ou plutôt littéraires?

3. Pour traduire une œuvre littéraire, le traducteur devrait-il essayer de rendre le style de l'original ? Le traducteur devrait-il essayer de se cacher pour représenter les caractères spécifiques de l'original?

4. La traduction littéraire devrait-elle transmettre les valeurs exotiques en maintenant leur étrangeté ou plutôt les naturaliser?

5. Que pensez-vous du point de vue sel on lequel la traduction littéraire devrait chercher la beauté plutôt que l'exactitude? 
6. Que pensez-vous du point de vue selon lequel la traduction en chinois d'une œuvre littéraire devrait employer le plus possible les expressions quadrisyllabiques, un moyen d'expression caractéristique du chinois?

7. La traduction littéraire devrait-elle faire prévaloir les avantages de la langue cible pour dépasser l'original?

8. Que pensez-vous du point de vue sel on lequel la traduction littéraire est une recréation dont le plus grand critère est la «naturalisation »?

9. Aimez-vous la traduction proche de la structure syntaxique de l'original ou plutôt celle qui, en changeant la structure syntaxique de l'original, s'exprime en chinois pur?

À ce questionnaire étaient joints des exemples caractéristiques extraits de cinq versions chinoises pour connaître le goût et la préférence personnelle des lecteurs.

Trois semaines après la publication du questionnaire, nous avions reçu 316 ré ponses de lecteurs d'âges et de niveaux culturels différents, de 30 régions chinoises, dont $70 \%$ ont fourni des réflexions centrées sur la traduction du Rouge et le N oir ; il est à noter que ces réponses n'étaient pas de simples oui ou non, mais au contraire, beaucoup d'entre elles étaient accompagnées de fines analyses et de riches réflexions instructives. Les réponses provenaient de milieux différents: étudiants, enseignants, employés, ouvriers, techniciens, ingénieurs, linguistes - par exemple Chen Yuan, un sociolinguiste très connu en Chine - , écrivains - comme Su Tong, auteur d'Épouses et concubines - , mais aussi traducteurs et théoriciens de la traduction.

Pour les lecteurs, la vogue actuelle de la retraduction des œuvres classiques étrangères en Chine est inévitable pour des raisons à la fois internes et externes.

Premièrement, c'est la nature de la traduction qui rend inévitable l'acte de retraduire. Selon la théorie herméneutique, tout texte est un système ouvert à ses lecteurs. En tant que «station de la circulation de la compréhension du texte original », la traduction est loin d'être une fin en soi, mais doit être un «texte scriptible» ouvert à ses lecteurs de langue d'arrivée et doit aussi attendre la prochaine interprétation, il est donc probable qu'en différentes époques paraissent différentes traductions d'une même œuvre. Deuxièmement, chaque traduction est en quelque sorte le produit d'une certaine époque et s'adresse aux lecteurs contemporains. Prenons un exemple ici tiré du Rouge et le Noir :

Combien de fois, songeant aux bals de Paris abandonnés la veille, et la poitrine appuyée contre ces grands blocs de pierre d'un beau gris tirant sur le bleu, mes regards ont plongé dans la vallée du Doubs! Au delà, sur la rive gauche, serpentent cing ou six vallées au fond desquelles I'œil distingue fort bien de petits ruisseaux. A près avoir couru de cascade en cascade on les voit tomber dans le D oubs. Le soleil est fort chaud dans ces montagnes; lorsqu'il brille d'aplomb, la rêverie du voyageur est abritée sur cette terrasse par de magnifiques platanes. (Imprimerie Tardy, 1958: 7)

多少时候, 我梦回昨宵遽然离弃巴黎的舞场, 我的胸怀斜倚着那光纹细耀的灰碧色 的巨石上, 我的目光投落在杜河的邱壑里！在那儿, 靠河岸所在左边, 蜿蜒着五六 重陵谷, 而在那些陵谷褶譬深处, 人们的眼睛可以分明地数得清许许多多细小的溪 流，人们可以看见这些小溪流流过一层一层的岩石，飞堕下来，成为一帘一帘的小 瀑布，终于倾落在杜河中，山间的阳光是很浱热的; 当日头垂直地照耀时，㮩息在 这片台阶上的游人的清梦遂为一列壮美的葆悬木所掩蔽。

(Traduction de Zhao Ruihong, 1944) 
有多少次我胸口靠在这些美丽的带点蓝色的灰色大块石头，心里想着头一天晚上在 巴黎放弃的舞会, 目光投向杜河河谷! 在那一边，河的岸上，有五六条弯弯曲曲的 山谷, 谷底的溪水清晰可辨, 它们形成一叠叠的瀑布倾泻下来以后, 注人杜河。在 这些山区里，太阳非常热; 每当烈日当空，在这片台阶上旅行者可以在高大悬铃木 的绿荫遮掩下遐想。

(Traduction de Hao Yun, 1990)

En comparant ces deux traductions chinoises, nous pouvons constater qu'elles sont, au niveau sémantique, fidèles au texte original, alors que les caractéristiques lexicales portent les traces du temps. Dans la première traduction, il y a des éléments linguistiques qui présentent un goût esthétique très apprécié peut-être des lecteurs anciens, mais qui ne se retrouvent plus dans le chinois moderne courant. En effet, au fil d'un demi-siècle, le goût des lecteurs a beaucoup évolué. La traduction a son âge. Si une traduction fourmille de mots désuets et a un style archaïque, elle ne peut correspondre au temps présent, et risque de tomber dans l'oubli et la disgrâce. Face à ce problème de vieillissement, on éprouve souvent le besoin de renouveler la traduction, surtout celle de grandes œuvres qui se sont taillé une réputation mondiale. Enfin, pour un traducteur qui travaille comme praticien bilingue (même multilingue), son savoir socioculturel et son expérience sont des éléments qui influencent sa traduction. Plus riches sont ses connaissances, meilleure sera sa traduction. C'est pourquoi il y a de nombreux traducteurs qui veulent retraduire les œuvres qu'ils avaient traduites antérieurement, comme Zhao Ruihong, premier traducteur chinois du Rouge et le Noir, âgé de plus de 80 ans, et qui se livre encore aujourd'hui à la «rénovation » de sa traduction qui date déjà d'un demi-siècle, et déclare: «En corrigeant les erreurs de I'ancienne traduction, je vais essayer de retraduire cette œuvre en un chinois plus simple et plus courant. »

M is à part les éléments internes de la traduction, les facteurs socio-économiques jouent aussi un rôle important dans cette vogue. Depuis l'adhésion de la Chine à la Convention de Berne, en 1992, toute publication d'œuvres étrangères contemporaines exige le versement de droits d'auteur, tandis que la traduction d'œuvres classiques n'est pas soumise à cette obligation. Contraints par des conditions financières, beaucoup d'éditeurs chinois ne peuvent que se tourner vers les œuvres classiques et organiser la retraduction d'œuvres très célèbres, mais déjà «âgées», pour combler, dans une certaine mesure, les manques de littérature étrangère sur le marché chinois.

Pour toutes ces raisons, la retraduction des œuvres classiques devient vraiment un «point chaud » parmi les phénomènes culturels en Chine actuelle. Selon les résultats de l'enquête sur la retraduction du Rouge et le Noir, les points les plus controversés sont centrés sur les problèmes suivants:

\section{Fidélité ou recréation}

Dans sa réponse, Hao Yun, un des traducteurs célèbres, déclare que ce qui est le plus important pour lui, c'est la fidélité à l'original, le traducteur n'a pas le droit de changer ce que l'auteur a créé dans son œuvre: «Si l'original est une algue, j'essaie de l'offrir avec son goût original aux lecteurs chinois, sans la changer en nouilles, bien que l'algue soit peut-être plus difficile à digérer que les nouilles pour les lecteurs. » 
Xu Yuanchong, un autre traducteur génial du Rouge et le Noir, préconise une attitude tout à fait différente de celle de Hao Yun. Selon lui, la traduction littéraire est en quelque sorte une concurrence de deux systèmes culturels et un art; et pour gagner, dans cette concurrence, il est important de faire prévaloir les avantages de la langue d'arrivée, c'est-à-dire de recréer l'original de sorte que la traduction le dépasse.

Voilà les deux opinions divergentes les plus typiques qui suscitent un débat acharné parmi les lecteurs. D'abord, qu'est-ce que la fidélité? Fidèle à quoi ? II y a tant d'éléments paradoxaux aux yeux du traducteur qu'il n'existe presque jamais de traduction absolument équivalente au texte original. «Quant à la recréation, elle serait aussi une sorte de fidélité, mais à un niveau plus élevé. Fidélité non seulement à l'esprit du texte, mais aussi au traducteur lui-même», écrit un lecteur dans sa réponse. Mais la plupart des lecteurs appuient un point de vue plus neutre et raisonnable: la traduction fidèle ne signifie pas nécessairement celle qui imite littéralement le texte d'origine. En tant que produit artistique et non absolument indépendant, elle doit posséder des éléments recréatifs, mais cette recréation a son degré inhérent et limité, c'est-à-dire que la traduction littéraire est une recréation restreinte du traducteur.

\section{Style de l'auteur et style du traducteur}

Si nous avions l'occasion de lire plus de trois traductions du Rouge et le N oir, nous aurions des impressions plus ou moins différentes sur le héros, Julien Sorel. C'est normal, car il y a autant de traducteurs que de façons d'interpréter le style de Stendhal. Parmi les 15 traducteurs de l'œuvre, on trouve des théoriciens de la traduction très célèbres qui ont chacun un avis personnel sur la pratique traduisante, et qui veulent l'affirmer dans leur propre texte traduit. Donc, bien qu'effacé, scribe de l'ombre et voué au silence, le traducteur est un écrivain autre et il a, de ce fait, une personnalité, un style. $M$ ais ici la question est de savoir quelle attitude on devrait adopter vis-à-vis de l'auteur et du traducteur.

Sur ce point, Su Tong, un des écrivains contemporains les plus connus en Chine, croit que le traducteur devrait respecter l'écrivain qu'il traduit et se garder de s'y substituer.

En principe, le traducteur doit observer une attitude objective et cacher le plus possible sa personnalité, [dit un lecteur] c'est-à-dire, qu'il doit essayer de pénétrer objectivement dans l'intention de l'auteur, et de faire sentir cette intention telle quelle aux lecteurs de la langue cible.

Un autre lecteur propose la notion de «points de fusion», basée sur l'identité des goûts personnels de l'auteur et du traducteur: «Ne voit-on pas qu'un auteur ne sera jamais moins trahi que lorsque toute son œuvre connaîtra la voix similaire traduisante?» Pour lui, il n'est de bonne traduction que s'il y a une affinité entre l'auteur et son traducteur, et ce qui est idéal pour un traducteur, c'est de choisir un écrivain dont le style est tout proche du sien.

\section{Traduction exotique et sino-centrique}

Une œuvre littéraire recèle toujours des valeurs culturelles qui se concrétisent dans la formulation linguistique, d'où l'étrangeté du texte, laquelle constitue pour beaucoup un des plus grands obstacles dans les activités traduisantes. 
«Je m'efforce toujours de traduire Le Rouge et le Noir en une langue purement chinoise», dit Xu Yuanchong, qui est pour la naturalisation des œuvres étrangères, dans la préface à sa traduction.

Mais est-ce que la traduction la plus naturalisée est celle qui est préférée du public chinois? La réponse est loin d'être affirmative selon les données de l'enquête. Pour beaucoup de lecteurs, il est préférable de maintenir l'étrangeté du texte d'origine car, par la lecture d'une œuvre traduite, on voudrait non seulement connaître I'histoire, mais aussi et surtout goûter l'exotisme et la sensualité propres à l'original.

La structure interne des phrases de l'œuvre française nous montre dans une certaine mesure la manière de penser française; si le traducteur la remplace selon son goût par l'expression purement chinoise (y compris les métaphores et les proverbes), beaucoup d'informations très intéressantes au niveau de la pensée et de la sémantique ainsi que des valeurs de formes au niveau esthétique risqueront de rester inconnues des lecteurs chinois, ce sera bien dommage!

Ce point de vue a certainement sa raison d'être, car avec la communication culturelle de plus en plus fréquente dans le monde, certaines structures et formulations caractéristiques du texte écrit en langue étrangère qui nous semblaient antérieurement incompréhensibles peuvent être introduites et assimilées par le chinois.

Bref, selon nos statistiques, $78,3 \%$ des lecteurs préfèrent une traduction ayant un goût exotique à une adaptation sino-centrique.

La floraison des versions chinoises d'une même œuvre offre l'embarras du choix aux lecteurs et représente aussi une lourde tâche pour la critique de la traduction.

«Faire ce que l'auteur a fait», voilà le critère traditionnel qui domine depuis longtemps la critique de la traduction. M ais dans I'histoire de la traduction, on n'a jamais trouvé un exemple qui puisse correspondre tout à fait à ce critère. Selon certains théoriciens de la traduction, c'est la différence des deux langues et des deux cultures en question qui crée l'impossibilité d'une traduction tout à fait fidèle, et le traducteur ne peut faire qu'un compromis entre des éléments paradoxaux. Ainsi, I'attitude raisonnable pour critiquer une traduction doit être plus objective en appliquant des principes plus souples, surtout sur différentes traductions d'une même œuvre, non seulement par le critère de la fidélité, mais aussi par le respect de l'activité subjective du traducteur. Citons un exemple tiré du Rouge et le Noir :

- Je ne suis pas du bois dont on fait les grands hommes, puisque je crains que huit années passées à me procurer du pain ne m'enlèvent cette énergie sublime qui fait faire les choses extraordinaires. (Imprimerie Tardy, 1958: 75)

$\mathrm{T} 1$ 我不是伟人的材料, 因为我害怕用来挣面包的八年时间从我这儿夺走使人做出非凡 事业的那种崇高的力量。

(Traduction de Hao Yun)

$\mathrm{T} 2$ 看来我不是成大人物的料, 花八年功夫混一口饭吃, 我都担心会壮志消尽, 无复行 非常之事的魄力了!

(Traduction de Luo Xinzhang)

Nous remarquons que les deux traductions sont fidèles au sens de l'original, mais ne sont pas tout à fait identiques l'une à l'autre. Les différences sont révélées surtout par leurs formulations linguistiques. D'abord, il y a une différence majeure au niveau syntaxique. Le traducteur 1 ( $\mathrm{H}$ ao Yun) transpose presque textuellement la structure 
compliquée de cette longue phrase sans changer l'ordre des mots, alors que le traducteur 2 (Luo Xinzhang), au contraire, la coupe en trois parties se conformant aux règles grammaticales traditionnelles du chinois écrit. On remarque aussi une différence au niveau lexical. Dans la traduction 1, le traducteur traduit explicitement «cette énergie sublime qui fait faire les choses extraordinaires» en conservant la couleur et le registre des mots, tandis que le traducteur 2 le rend implicitement par un chinois élégant, dans un style qui nous semble très soigné.

Voyons encore les opinions de ces deux traducteurs sur leur méthode de traduction:

H ao Yun dit transposer non seulement le sens, mais aussi la structure des phrases qui pourrait donner sans doute une sensation de nouveauté aux lecteurs chinois. II espère ainsi avoir une traduction moins dénaturée par rapport à l'original.

Pour Luo Xinzhang, ce qui importe le plus c'est un style classique et concis digne de celui de Stendhal. II essaie d'offrir une traduction plus lisible pour le public chinois, si l'on veut, un vrai produit littéraire.

Ayant pour but de faire connaître la façon de s'exprimer des Français et leurs valeurs exotiques, $\mathrm{H}$ ao Yun a essayé de créer une atmosphère exotique dans sa traduction, qui est considérée commel'exemple de la traduction «équivalente», et, du point de vue esthétique, il est évident que la traduction de Luo est beaucoup plus littéraire et éégante, étant un produit recréé, un vrai texte. Ces deux traductions ont chacune leurs points forts et sont appréciées par des lecteurs de milieux différents. Ce qui explique que les deux traductions ont connu chacune une large diffusion et un très gros tirage.

En ce qui concerne la critique, les critères d'évaluation d'une traduction ne devraient pas être absolus. On doit tenir compte de la textualité de l'original et de la productivité du traducteur, et aussi du goût des lecteurs. La fonction de la critique n'est pas de définir des critères formels de traduction, mais plutôt d'aider les lecteurs à bien choisir ce qu'ils préfèrent en se référant à des facteurs extra-linguistiques et socioculturels. Et, selon Antoine Berman, la critique de la traduction a pour fonction essentielle d'ouvrir de nouvelles possibilités à la retraduction. L'évaluation de la traduction devrait être fondée sur un double critère: la poéticité et l'éthicité, la première résidant «en ce que la traduction a réalisé un véritable travail textuel, a fait texte, en correspondance plus ou moins étroite avec la textualité de l'original »; la deuxième résidant «dans le respect, ou plutôt, dans un certain respect de l'original », commel'a proposé Berman (1995: 92).

Comme nous venons de le montrer, dans le débat sur la retraduction du Rouge et le $\mathrm{N}$ oir, des problèmes fondamentaux de traduction ont été soulevés; ils concernent le but fixé, la nature de la traduction, la tâche du traducteur, les rapports entre l'auteur, le traducteur et le lecteur, l'historicité du texte traduit, les éléments linguistiques et extra-linguistiques, les facteurs socioculturels, idéologiques, etc. Avec l'opposition très souvent dualiste entre la traduction littérale et la traduction libre, la forme et le fond, la fidélité et la trahison, il est peut-être impossible de trouver une solution acceptable pour tous aux problèmes posés. Cette opposition est-elle un dilemme éternel dont les traducteurs et les théoriciens ne pourront jamais sortir? 


\section{UNE PROPOSITION}

De ce que nous avons exposé, on peut tirer au moins deux conclusions. La première est que la pratique traduisante pose dès le début des problèmes fondamentaux aux traducteurs. Ils cherchent toujours à y apporter une certaine solution, en fonction d'une certaine conception ou d'une perception du traduire, de son sens, de ses finalités. La deuxième est que la façon traditionnelle d'aborder les problèmes relatifs à la traduction nous mène toujours à une opposition dualiste fondée sur la conception simpliste de la langue qui cache plutôt le fond des problèmes. Selon nous, une théorie de la traduction n'a pas de raison d'être si elle ne peut que perpétuer cette opposition dualiste et si elle végète dans des réflexions empiriques. II est donc nécessaire d'aborder ces problèmes par d'autres approches et de fonder une théorie de la traduction qui ne soit pas «activité spéculative mais [... ] pratique théorique, pour la connaissance historique du processus social de textualisation», comme l'a proposé Henri Meschonnic (1973). Les problèmes les plus controversés dans le débat sur la retraduction du Rouge et le Noir en Chine justifient dans une large mesure le bien-fondé des propositions de Meschonnic. Selon lui (1973: 340):

La «langue» - la «littérature»; ou la langue-culture, ou le sens - la forme; il n'y a pas deux choses dissociables, hétérogènes. Quand il y a un texte, il y a un tout, traduisible comme tout. La pratique et I'histoire de la traduction le montre. Ni la théorie de la communication, ni une linguistique de la traduction telle qu'elle utilise la grammaire transformationnelle et la sémantique structurale ne peuvent en rendre compte, car ce sont des conceptualisations dualistes. Seule une poétique de la traduction peut théoriser le succès ou l'échec des traductions.

En effet, la conception d'Henri M eschonnic ne sépare pas l'écriture et la traduction dans leur histoire et dans leur théorie. Elle peut nous aider à répondre à certains problèmes posés dans le débat sur la retraduction du Rouge et le $\mathrm{N}$ oir. Dans un article intitulé "De la poétique de la traduction ${ }^{3}$ ", M lle Yuan Xiaoyi et moi-même avons essayé d'analyser les 36 propositions de M eschonnic, et ses contributions à la théorie de la traduction se sont révélées très intéressantes. Au lieu de traiter de son apport théorique aux études des problèmes fondamentaux de la traduction, nous voulons présenter une proposition pour une étude des niveaux de la traduction.

Du point de vue de la stratification, toute entité organique est constituée selon un certain ordre et une certaine hiérarchie. Cette entité est, dans une certaine mesure, un système formé par un nombre déterminé d'éléments interactifs, et la structure interne de chaque système est stratifiée. La traduction en tant qu'activité ne fait pas exception. Elle s'avère aussi un système. Elle possède ses propres éléments tels que le contenu, la forme, la finalité, etc. L'évolution de ces éléments, leur indépendance relative et leur interaction constituent la base objective de l'existence des niveaux de la traduction.

Selon Antoine Berman, tout traducteur entretient un rapport spécifique avec sa propre activité. Les conceptions et les perceptions de la nature du traduire sont loin d'être convergentes. Cela est dû, d'une part, à la complexité de l'activité de traduction et, d'autre part, à la sous-estimation de l'acte de traduire dans les réflexions théoriques. On a souvent tendance à ne voir qu'un aspect du traduire ou à opposer ses éléments interdépendants. La conclusion tirée de cette conception partielle ou dualiste conduit fatalement à négliger certains éléments existant à un niveau différent. 
Par exemple, ceux qui considèrent la traduction comme une opération purement linguistique veulent toujours isoler l'opération traduisante dans la sphère linguistique et placer leurs recherches théoriques sous l'égide de la linguistique. Or, l'activité traduisante, quand on recense tous ses aspects, dans toute sa complexité, ne se limite pas à la seule opération linguistique. Et les problèmes posés par la traduction de divers genres ne peuvent être résolus uniquement à la lumière des techniques d'analyse actuellement à I'honneur en linguistique. II en est de même de la conception artistique de la traduction. En apparence, la traduction littéraire est caractérisée essentiellement par la recréation de formes artistiques reflétant les mêmes effets que l'œuvre originale. Or les effets artistiques ne peuvent se séparer nettement des formes et des moyens d'expression d'une langue. Cette recréation est en réalité limitée par son caractère particulier de convergence linguistique, d'autant plus que la traduction met nécessairement en jeu non «seulement le vocabulaire, mais aussi la syntaxe, ainsi que la stylistique et la dimension proprement idiomatique des langues concernées» (Ladmiral 1979: 16). En fait, les problèmes posés dans la pratique de la traduction ne sont ni purement linguistiques ni purement artistiques. Et les solutions qu'on essaie d'apporter aux divers problèmes posés à la traduction ne peuvent, par voie de conséquence, être trouvées que lorsqu'on connaît les lois régissant les différents niveaux de l'activité traduisante. Toute conception simpliste et partielle de la traduction ne peut évidemment pas nous aider à trouver des réponses ni aux problèmes théoriques ni aux innombrables problèmes concrets que ne cesse de nous poser la pratique de la traduction. II est donc nécessaire d'étudier les différents aspects de la traduction et ensuite de classer de façon objective les différents niveaux de l'activité traduisante.

Les points de vue les plus représentatifs sur la nature de la traduction se contre disent aussi souvent que possible. Cette contradiction résulte dans une large mesure du fait qu'on n'a pas de vue d'ensemble de la conception de la traduction. Quand on parle de la traduction que nous entendons ici dans son sens dynamique, c'est-à-dire l'activité du traducteur, on commence assez souvent par se demander comment la traduction peut se réaliser puisqu'il existe bel et bien une différence systématique entre les langues concernées. Pour traduire, il faut tout de même un terrain commun aux langues en question. Quel est ce terrain commun à partir duquel on peut procéder au transfert en des langues différentes? Voilà une question fondamentale à laquelle aucune étude théorique de la traduction ne peut éviter de répondre. Logiquement parlant, ce terrain commun qui pourrait servir de base à l'activité traduisante doit en premier lieu posséder un caractère universel, car sans cette universalité qui existe à un niveau profond et commun à toutes les langues du monde, la traduction entre langues différentes ne peut se réaliser. Et ce terrain commun, s'il existe, constitue pour ainsi dire le niveau de base de la traduction.

Remarquons ensuite que toute activité traduisante met inévitablement en jeu les structures et les formes de la langue; or, personne n'ignore que ces structures et ces formes se caractérisent essentiellement par la nationalité et l'arbitraire. Un transfert de forme à forme n'a pas une finalité en soi. Alors, une autre question se pose: Qu'est-ce qu'on traduit, exactement? Depuis ces dernières années, on commence à s'accorder pour penser que la traduction, désignant «toute forme de médiation interlinguistique», a pour tâche de faire passer un message d'une langue de départ dans une langue d'arrivée, permettant ainsi de transmettre de l'information entre les locuteurs de langues différentes. Justement, les structures et les formes de la langue n'ont 
de raison d'être que par leur fonction de réalisation de la pensée humaine et de transmission de l'information entre les hommes parlant une même langue ou une langue différente. Pour transmettre l'information que recèlent les structures et les formes, force nous est de partir du sens. Et, à ce niveau de la traduction, on devrait essayer d'étudier les lois régissant le maniement des significations et la transmission sémantique.

II est incontestable que la transmission sémantique constitue la tâche principale de la traduction. Cependant, en considérant les fonctions de la langue, nous pouvons tout de suite constater que la transmission esthétique ne peut non plus être négligée dans la traduction, surtout littéraire. La transmission esthétique - et j'entends ici par la reproduction de la beauté artistique de l'œuvre originale - pose au traducteur des obstacles beaucoup plus difficiles à surmonter que la transmission sémantique, parce qu'au niveau esthétique, la beauté se trouve non seulement sur le plan de la pensée, mais aussi sur le plan de la forme de l'expression ; et aucun traducteur sérieux ne peut fuir les obstacles qu'on rencontre inévitablement quand on essaie de transférer les effets esthétiques produits par les formes littéraires. Quels sont ces obstacles particuliers? Comment les surmonter? Ces questions nous amèneraient à traiter des problèmes existant à ce niveau.

Comme nous venons de le montrer assez sommairement, la traduction est une activité à plusieurs niveaux et, à chaque niveau, des problèmes particuliers existent, qu'on néglige bien souvent dans la pratique traduisante. En tenant compte des éléments de la traduction, des fonctions des signes linguistiques, de leur interdépendance et de leur interaction, nous suggérons la classification suivante de la traduction: niveau de pensée, niveau sémantique et niveau esthétique. À partir de cette classification, si nous regardons de plus près ces trois niveaux et analysons leurs relations, leurs points communs et leurs particularités, nous pouvons voir plus clairement les obstacles de chaque niveau, les lois régissant la traduction à chaque niveau et ce qui permettrait de proposer des principes applicables dans la pratique traduisante.

Avec cette conception de la stratification de la traduction, la traduction religieuse et la traduction littéraire ne seront plus opposées, car ces deux types de traduction présentent des problèmes communs au niveau de la pensée et au niveau sémantique. L'opposition entre la forme et le fond, I'objectivité du texteà traduire et la subjectivité du traducteur cédera la place à une dialectique selon laquelle la traduction d'un texte devrait être structurée comme un texte dans son unité.

Pour nous, la traduction n'est pas celle de la langue seule, mais un chemin qui ouvre l'accès authentique à une pensée, un cas particulier de communication interlinguistique, interculturelle et interlittéraire, et un mode de textualisation. Le rapport qui lie une traduction à son original est unique en son genre, mais varié selon les cas, car les facteurs qui agissent dans le processus de la traduction s'avèrent divers. Les relations entre les différents niveaux se caractérisent par l'interdépendance et la complémentarité, mais chaque niveau a ses caractéristiques propres, ses problèmes propres, et demande donc une opération appropriée à sa nature. Une connaissance profonde et objective des relations entre les différents niveaux, de leurs caractères spécifiques et de leurs problèmes particuliers peut au moins nous aider à bien mesurer les possibilités et les limites de la traduction; et contribuer à une orientation moins aveugle de la pratique en essayant de rendre l'opération traduisante harmonieuse sans aboutir à des extrêmes. 
Traduire une œuvre, c'est en réalité chercher l'équivalence dans la différence. La différence se trouve à tous les niveaux, mais dans des proportions inégales, ce qui fait qu'au niveau fondamental qui se base sur l'universalité de la pensée, on peut espérer une équivalence à peu près identique, et au niveau sémantique où l'on doit obéir aux lois et règles de chaque langue, les possibilités d'une correspondance se trouvent inévitablement réduites, et on ne peut qu'essayer de chercher une équivalence dynamique, c'est-à-dire une équivalence qui ne se limite pas au plan formel, mais touche aussi les plans sémantique et pragmatique. Au niveau esthétique, qui se caractérise essentiellement par la subjectivité, l'affectivité et la créativité, la reproduction des valeurs ou effets esthétiques et du pouvoir créatif langagier des traducteurs, par conséquent, le degré d'équivalence varie souvent d'une personne à l'autre et il n'y a donc rien d'étonnant qu'à ce niveau de traduction on parle de recréation ou de cocréation du traducteur.

Qui dit différence, dit obstacles. C'est ainsi que nous proposons une étude, bien que partielle, des principaux obstacles ainsi que de leurs causes et de leurs conséquences. Dans la pratique de la traduction, on a souvent tendance soit à minimiser la différence et les obstacles qu'elle entraîne, soit à exagérer les difficultés qui proviennent de tous les niveaux de la traduction. Ces deux attitudes mènent à une conception simpliste de la traduction: tout ou rien. Seule une étude approfondie et scientifique des obstacles peut nous permettre d'établir une théorie correcte de la possibilité de traduire. C'est sur la base d'une analyse objective que nous pouvons envisager les méthodes appropriées à chaque catégorie de problèmes. Le choix de méthodes ou de procédés ne dépend pas, contrairement à ce que l'on croit, de la volonté du traducteur, mais se trouve déterminé par les caractéristiques propres à chaque niveau de la traduction et par les facteurs qui influencent l'acte de traduire, comme les facteurs historiques, sociaux, littéraires, idéologiques, etc. Ainsi, qu'il s'agisse de méthodes directes ou indirectes, il n'y a pas de différence, bonne ou mauvaise.

Nous croyons pouvoir apporter à la critique de la traduction un certain crité rium, en distinguant trois niveaux de traduction et en examinant leurs relations. L'harmonie de ces trois niveaux constitue une condition indispensable à une bonne traduction. Ainsi, juger ou critiquer une œuvre de traduction implique une vue d'ensemble. Et, vue sous cet angle, la polémique sur la fidélité au contenu ou à la forme n'aurait plus sa raison d'être, parce que toute traduction n'opère pas seulement sur le plan du contenu ou de la forme, le contenu et la forme n'étant pas fondamentalement incompatibles. Une pleine connaissance des caractéristiques des signes linguistiques peut nous aider à résoudre de façon convenable et acceptable la contradiction qu'ils constituent. La recherche d'une équivalence des effets ou des valeurs fonctionnelles est en quelque sorte une tentative de transformation des formes linguistiques.

Comme on le voit, notre but n'est pas de dresser ici un bilan des éléments d'une science de la traduction, mais d'ouvrir un domaine ou un nouvel angle de réflexion sur la nature et le processus de la traduction, et de mesurer objectivement la complexité et la tâche de la traduction pour qu'on ait une conscience plus juste des problèmes fondamentaux du traduire. À notre avis, une vraie science de la traduction doit avoir un double sens: science prenant le savoir de la traduction comme objet et science guidant la pratique de la traduction. 
6o Meta, XLIV, 1, 1999

\section{NOTES}

1. Lire, n 252, février 1997, p. 46.

2. Recueil d'études sur la traduction (1949-1953), textes rassemblés par la M aison d'édition de didactique des langues et recherche, Beijing, 1984, p. 80.

3. Yuan Xiaoyi et Xu Jun (1995) : «De la poétique de la traduction», Études des langues étrangères, $n^{\text {os }} 3-4$, Nanjing, Chine.

\section{RÉFÉRENCES}

Ballard, Michel (1995): De Cicéron à Benjamin, traducteurs, traductions et réflexions, Lille, Presses Universitaires de Lille.

Ber man, Antoine (1984) : L'Épreuve de l'étranger, culture et traduction dans l'Allemagne romantique, Paris, Gallimard.

Ber man, Antoine (1995) : Pour une critique des traductions: John Donne, Paris, Gallimard.

Cary, Edmond (1963) : Les Grands Traducteurs français, Genève, Georg.

Ch en Fukang (1992) : H istoire des études théoriques de la traduction en Chine, Éditions de l'Enseignement de langues étrangères de Shanghai.

Dol et, Étienne [1540] (1972): La Manière de bien traduire d'une langue en aultre, Genève, Slatkine.

Ladmir al , J.-R. (1979) : Traduire: théorèmes pour la traduction, Paris, Petite Bibliothèque Payot. M esch on nic, Henri (1973) : Pour la poétique II, Paris, Gallimard. 